\title{
UTILIZAÇÃO DE SUBPRODUTOS AGROINDUSTRIAIS NA PRODUÇÃO DE BRIQUETES
}

\author{
USE OF AGROINDUSTRIAL BY-PRODUCTS IN THE PRODUCTION OF \\ BRIQUETTES \&
}
UTILIZACIÓN DE SUBPRODUCTOS AGROINDUSTRIALES EN LA PRODUCCIÓN DE BRIQUETAS \&

Recebido em: 26/06/2020 - Aprovado em: 26/09/2020 - Publicado em: 20/04/2021

doi) http://dx.doi.org/10.18011/bioeng2021v15n1p1-26

Jaqueline Zanovelli Nalevaiko ${ }^{1}$ (jaquee_zn@hotmail.com)

Paulo André Cremonez' ${ }^{1}$ (pa.cremonez@gmail.com)

Joel Gustavo Teleken ${ }^{1}$ (joel.teleken@ufpr.br)

1 Universidade Federal do Paraná. Palotina, PR, Brasil.

\section{RESUMO}

O atual cenário energético mundial é de busca por combustíveis mais limpos e novas fontes de energia renováveis, com destaque à utilização de biomassa residual. $O$ presente trabalho busca avaliar as características físico-químicas de briquetes produzidos a partir do bagaço da cana-de-açúcar, bagaço de mandioca e cama de aviário. Os subprodutos passaram por análises antes e após a briquetação. Foram produzidos briquetes com diferentes percentuais de mistura (M1, M2 e M3). Os briquetes produzidos apresentaram valores de eficiência energética, resistência mecânica e densidade aparente e energética coerentes com valores da literatura e de normativas internacionais. Em relação ao teor de cinzas nenhum deles se enquadra nos parâmetros europeus de fabricação de briquetes. A mistura com melhor eficiência energética foi M2, com poder calorífico de 16,41 MJ/kg e também o menor teor de cinzas, de 7,96\%, e melhor relação $\mathrm{C} / \mathrm{H}$, porcentagem de hidrogênio e de carbono, sendo assim o de maior potencial energético de queima. A utilização de briquetes produzidos a partir de biomassa residual apresenta muitas vantagens, contribuindo em aspectos econômicos, sociais e ambientais.

Palavras-chave: Energia. Biocombustível Sólido. Biomassa Residual. 


\section{INTRODUÇÃO}

Os problemas ambientais da atualidade, causados em grande parte pelas emissões de Gases do Efeito Estufa (GEE's) da queima de combustíveis fósseis e o crescente preço do petróleo, abrem espaço para uma busca por fontes de energia renováveis e por combustíveis mais limpos, com a intenção de garantir o desenvolvimento econômico sem que ocorra uma agressão maior ao meio ambiente e à saúde humana (ALI et al., 2019; BORGES et al., 2016; KIM et al., 2016).

Dentre as alternativas, destaca-se a utilização da biomassa como fonte de energia primária e renovável, seja do ponto de vista ambiental ou econômico. A biomassa, recurso obtido através da matéria orgânica pode ser convertido em combustível gasoso, líquido ou sólido, e seu emprego na produção de energia apresenta grande potencial de crescimento uma vez que sua disponibilidade é abundante no país (EPE, 2020).

Segundo a Agência Internacional de Energia (AIE, 2015), 41\% da eletricidade do mundo decorre da queima do carvão. A utilização de tecnologias limpas de combustíveis sólidos é a abertura para a substituição do uso do carvão, dentre eles ganham notoriedade os pellets, briquetes, lascas de madeira, madeira e biocarvão (BONASSA et al., 2016).

As fontes primárias consideradas para a produção descentralizada dos biocombustíveis sólidos são os resíduos agrícolas, e tais produtos podem ser utilizados para cogeração de energia elétrica e térmica, queima junto com outros combustíveis ou simplesmente em combustão direta para geração de calor, suprindo necessidades de diversas atividades humanas e reduzindo grande parcela de impactos ambientais (SAWIN et al., 2012).

Segundo a Empresa de Pesquisa Energética (EPE) nos próximos anos os biocombustíveis sólidos residuais terão elevação de utilização, substituindo a lenha nos setores agropecuário e industrial, como consequência de um aumento do custo da lenha e de uma redução de custos dos biocombustíveis sólidos. O potencial de produção pode ser mais relevante para a matriz energética brasileira caso o cenário seja favorável para a bioenergia e oferta descentralizada de energia (EPE, 2016).

De acordo com Embrapa Agroenergia (2015) os briquetes podem ser produzidos a partir de diversos resíduos vegetais, tendo diâmetro superior a $50 \mathrm{~mm}$. A compactação desses resíduos agrícolas ou florestais traz vantagens na parte operacional, logística, ambiental e energética. 
Destaca-se no estado do Paraná, dentre os resíduos agroindustriais, aqueles oriundos das usinas de açúcar, amido de mandioca e avicultura, por conta de sua grande quantidade de produção (IBGE, 2017).

Das usinas de açúcar os principais resíduos são o bagaço e a palha da cana, com elevado potencial de aproveitamento energético. Aproximadamente um terço da energia da cana-de-açúcar está armazenada no caldo em forma de açúcares, sendo que o restante é armazenado na biomassa (bagaço e palha) (GOES, 2008).

No processamento da mandioca, além da extração do amido são gerados resíduos como a manipueira e o bagaço em volumes significativos. $O$ bagaço apresenta características fibrosas, e quando seco apresenta teores de amido superiores a $50 \%(\mathrm{~m} / \mathrm{m})$, além de elevadas concentrações de fibras, que são compostas de grande variedade de substâncias que apresentam distintas propriedades físicas, químicas e fisiológicas (ZENATTI et al., 2015).

$\mathrm{Na}$ avicultura, setor agroindustrial de suma importância comercial e alimentícia, são geradas grandes quantidades de resíduos, sendo a cama de aviário o rejeito de maior volume. Para muitos produtores os problemas com a gestão de resíduos são bastante evidentes, pois os mesmos podem causar odores desagradáveis e causar poluição por lixiviação e erosão quando localizados próximos a cursos d'água (MONTAGNA, 2017).

A busca por alternativas de destinação final é de grande importância para o desenvolvimento sustentável desses setores. Sua utilização na briquetagem apresenta vantagens para sua estocagem e transporte, além da geração de energia. No processo de briquetagem a biomassa é submetida a elevadas temperatura e pressão, não alterando a constituição química, porém aumentando sua densidade e energia por m3 de biomassa (COSTA, 2019).

Com base nas informações anteriormente apresentadas, o presente trabalho ${ }^{1}$ tem como objetivo avaliar o potencial energético da biomassa do bagaço da cana-de-açúcar, bagaço de mandioca e cama de aviário, separadamente ou em diferentes razões de misturas, na produção de briquetes. Pretende-se ainda avaliar o potencial energético e as características físico-químicas da biomassa e dos briquetes produzidos

\footnotetext{
1 Trabalho baseado na Dissertação de Mestrado intitulada "Produção de briquetes a partir de coprodutos agroindustriais: bagaço de cana de açúcar, cama de aviário e resíduo de aminodaria”. Disponível em: https://acervodigital.ufpr.br/bitstream/handle/1884/65572/R\%20-\%20D\%20-

\%20VENICIO\%20STEFFLER.pdf?sequence=1\&isAllowed=y. Acesso em: 14/04/2021.
} 


\section{MATERIAIS E MÉTODOS}

As biomassas utilizadas foram doadas por indústrias das regiões Oeste e Noroeste do Paraná. Foram coletas aproximadamente $500 \mathrm{~kg}$ de cada biomassa a ser analisada: bagaço de cana-de-açúcar, bagaço de mandioca e cama de aviário.

Após a coleta as amostras foram secas em estufas de secagem com circulação e renovação de ar. As amostras de bagaço apresentaram teor de umidade acima de $85 \%$. Como pré-tratamento fez-se remoção de parte da umidade por centrifugação do material até atingir aproximadamente $35 \%$ de umidade, para então serem levadas às estufas.

\subsection{ANÁLISES IMEDIATA E ELEMENTAR DOS COPRODUTOS E BRIQUETES}

A determinação dos teores de umidade, materiais voláteis, carbono fixo e cinzas para as biomassas e os briquetes foi realizado pelo método ASTM (E-870-82), empregado para análise imediata de biomassa e resíduos. As análises foram realizadas em triplicata.

Para estimar os teores de Carbono, Hidrogênio e Oxigênio foram utilizadas Equações para determinação das composições por correlação (1, 2 e 3) apresentadas abaixo (PARIKH et al., 2007).

$$
\begin{aligned}
& C=0.637 C F(\%)+0.455 M C(\%) \\
& H=0.052 C F(\%)+0.062 M V(\%) \\
& O=0.304 C F(\%)+0.476 M V(\%)
\end{aligned}
$$

Onde:

C: teor de carbono (\%);

CF: carbono fixo (\%);

MC: massa de cinzas (\%);

$\mathrm{H}$ : teor de hidrogênio (\%);

MV: materiais voláteis (\%);

O: teor de oxigênio (\%). 


\subsection{DETERMINAÇÃO DO PODER CALORÍFICO}

O poder calorífico superior (PCS) das amostras foi estimado através da Equação 4, proposta por Parikh et al. (2004).

$P C S=0.3536 C F(\%)+0.1559 M V(\%)-0.0078 C Z(\%)(4)$

Onde:

PCS: poder calorífico superior $(\mathrm{MJ} / \mathrm{kg})$;

CF: carbono fixo (\%);

MV: materiais voláteis (\%);

CZ: cinzas (\%).

Para determinação do poder calorífico inferior $(\mathrm{PCl})$, é necessário conhecer teores de carbono e hidrogênio da amostra e aplica-los na Equação 5, proposta por Parikh et al. (2004).

$$
P C I=\left(P C S-\frac{C(\%) .600 .9 . H(\%)}{100}\right) / 239
$$

Onde:

PCI: poder calorifico inferior $(\mathrm{MJ} / \mathrm{kg})$;

PCS: poder calorifico superior (MJ/kg);

C: carbono (\%);

$\mathrm{H}$ : hidrogênio (\%).

Para determinação do poder calorífico útil (PCU) de uma biomassa, segundo o mesmo autor, é necessário conhecer o teor de umidade da amostra e seu poder calorífico inferior. Aplicando-se a Equação 6 obtém-se o valor de PCU em MJ/kg.

$$
P C U=P C I(1-0,01 \cdot U(\%))-(600.0,01 \cdot U(\%)) / 239
$$

Onde:

PCU: poder calorífico útil (MJ/kg);

PCl: poder calorífico inferior (MJ/kg);

U: umidade (\%). 


\subsection{BRIQUETAGEM}

As misturas 1, 2 e 3 foram moídas em um moinho industrial de facas com peneira abertura de $10 \mathrm{~mm}$ e em seguida briquetadas. Os briquetes foram produzidos na empresa BIOMASSA localizada no município de Guarapuava - PR.

A briquetadeira utilizada é do tipo rosca-sem-fim, e seu motor tem potência de $50 \mathrm{cv}$ alimentado a diesel. Possui capacidade de fornecer 80 toneladas-força (tnf) e calor de 7200 Watts (W) através do eletrodo UTP Ducsteno 65 fixado na parte externa do canhão, o qual possui resistência de oxidação até $900^{\circ} \mathrm{C}$, dureza de aproximadamente $240 \mathrm{HB}$, corrente contínua ou alternada (70 A até $100 \mathrm{~A})$.

\subsection{DETERMINADAÇÃO DA DENSIDADE DOS BRIQUETES}

A densidade dos briquetes foi determinada pelo método estereométrico, adaptado de Protásio (2011), utilizando-se a Equação 7.

$d=m /\left(V_{T O T A L}-V_{\text {INTERNO }}\right)$

Onde:

$\mathrm{d}$ : densidade $\left(\mathrm{kg} / \mathrm{m}^{3}\right)$;

m: massa (kg);

VTOTAL: volume total $\left(\mathrm{m}^{3}\right)$;

VINTERNO: Volume Interno $\left(\mathrm{m}^{3}\right)$.

Para determinação do volume do briquete foi considerado sua forma cilíndrica. A massa foi obtida em balança digital Shimadzu, modelo BL $3200 \mathrm{H}$.

A densidade energética foi calculada multiplicando-se o poder calorifico superior dos briquetes, pelos seus valores de densidade aparente.

\subsection{ANÁLISE DE COMPRESSÃO DOS BRIQUETES PRODUZIDOS}

O valor da resistência à compressão axial foi determinado por meio da relação entre a área de cada briquete e sua resistência. $O$ equipamento aplica uma força perpendicular ao comprimento do briquete por meio de um pistão, com velocidade de ensaio pré- 
estabelecida e determina a força da resistência desse material até a sua ruptura gerada por uma carga de tensão.

Utilizou-se uma máquina de ensaio universal modelo $41 \mathrm{AGX}$, Shimadzu, de $100 \mathrm{kN}$, do Laboratório de Física da Unicentro (CEDETEG). A velocidade de carregamento aplicada aos corpos de prova foi de $5 \mathrm{~mm} / \mathrm{min}$. O resultado de resistência à compressão foi determinado por um software acoplado ao equipamento. O procedimento de análise foi realizado de acordo com a metodologia NBR 5739 - Ensaio de compressão de corpo cilíndrico (ABNT, 2018), uma vez que não se tem normas específicas para testes em briquetes dessa natureza.

\subsection{PLANEJAMENTO EXPERIMENTAL E ANÁLISE ESTATÍSTICA}

O planejamento experimental foi feito com base nos resultados encontrados na análise imediata, análise elementar e poder calorífico dos resíduos in natura. Foram determinadas restrições para definir os valores percentuais das misturas das biomassas para produção de briquetes e as mesmas são apresentadas na Tabela 1.

Tabela 1 - Restrições dos valores de biomassa em base mássica.

\begin{tabular}{ccc}
\hline Bagaço de Cana & Bagaço de Mandioca & Cama de Aviário \\
\hline $30 \%>X>100 \%$ & $5 \%>X>15 \%$ & $30 \%>X>100 \%$ \\
\hline
\end{tabular}

Os valores percentuais foram definidos com base nas características de cada biomassa. O bagaço de cana e a cama de aviário, tiveram um range de mistura maior, quando comparado ao bagaço de mandioca por apresentarem menor umidade quando in natura. O bagaço de mandioca, apresentou teor de umidade in natura superior a $85 \%$, dificultando sua utilização. Por outro lado, o bagaço de mandioca é rico em amido, sendo de grande importância na produção dos briquetes atuando como material aglutinante. Os percentuais de misturas utilizados são apresentados na Tabela 2.

Tabela 2 - Percentuais de biomassas utilizadas em base mássica.

\begin{tabular}{cccc}
\hline Mistura & Bagaço de cana & Bagaço de mandioca & Cama de aviário \\
\hline M1 & 30 & 5 & 65 \\
M2 & 42,5 & 15 & 42,5 \\
M3 & 65 & 5 & 30 \\
\hline
\end{tabular}

Fonte: Os autores, 2020. 
O experimento foi avaliado em delineamento inteiramente casualizado (DIC), utilizando 3 tratamentos (tipo de briquete) e 3 repetições para cada variável estudada. Para verificação de diferenças estatísticas entre as amostras, os dados obtidos foram submetidos ao Teste F por meio da análise de variância (ANOVA). Quando identificadas diferenças significativas na ANOVA, aplicou-se o teste de Tukey ao nível de $5 \%$ de significância. Os dados foram analisados com o auxílio do software SPSS, versão 20.0.

\section{RESULTADOS E DISCUSSÕES}

\subsection{DETERMINAÇÃO DO TEOR DE UMIDADE DOS COPRODUTOS IN NATURA}

O teor de umidade é um fator de grande importância no uso da biomassa como combustível, pois apresenta uma relação inversa com o poder calorífico, prejudica o armazenamento, uma vez que pode ocasionar a proliferação de fungos e a degradação do material, além de elevar os custos de transporte.

O bagaço de mandioca apresentou teor de umidade de $90,41 \%$, o bagaço de canade-açúcar apresentou teor de $46,17 \%$, e a cama de aviário apresentou um baixo teor de umidade, de 17,84\%, favorável para a produção de briquetes.

\subsection{ANÁLISE IMEDIATA E ELEMENTAR DOS COPRODUTOS APÓS SECAGEM}

$\mathrm{Na}$ Tabela 3 visualizam-se os teores de umidade, carbono fixo, materiais voláteis e cinzas para bagaço de cana-de-açúcar (BC), bagaço de mandioca $(\mathrm{BM})$ e cama de aviário (CA).

Tabela 3 - Análise imediata das amostras.

\begin{tabular}{ccccc}
\hline Item & Umidade (\%) & Carbono Fixo (\%) & Materiais Voláteis (\%) & Cinzas (\%) \\
\hline BC & 5,42 & 13,96 & 80,98 & 5,06 \\
BM & 11,30 & 16,88 & 81,68 & 1,44 \\
CA & 1,34 & 15,88 & 64,07 & 20,05 \\
\hline
\end{tabular}

Fonte: Os autores, 2020.

Os resultados obtidos para bagaço de mandioca foram de mesma ordem de grandeza dos obtidos por Pattiya et al. (2010), que relata valores de 8,3\% para umidade, $3,7 \%$ para o teor de cinzas, $16,7 \%$ para carbono fixo e $71,3 \%$ para material volátil. Já para 
Zanatta (2012), os valores encontrados foram 7,6\% para umidade, $81,7 \%$ para teor de voláteis, $9,7 \%$ teor de carbono fixo e $1,0 \%$ para o teor de cinzas. O teor de cinzas encontrado na biomassa é considerado excelente para combustão do material.

Os resultados para ao bagaço de cana-de-açúcar obtidos foram de mesma ordem de grandeza dos obtidos por Zanatta (2012), que verificou valor de 7,2\% para umidade, $82,5 \%$ para materiais voláteis, 9,3\% para teor de carbono fixo e $0,9 \%$ teor de cinzas. Já Brand (2010), encontrou 14,95\% para teor de carbono fixo, 11,27\% para teor de cinzas, e $73,78 \%$ para voláteis. Em relação a umidade, ela depende muito do tempo de exposição, coleta e armazenamento do bagaço de cana-de-açúcar para análise. As variações encontradas se devem as diferentes condições climáticas e cultivos da cultura.

Segundo Paula et al. (2011) a análise do teor de cinzas nos combustíveis de biomassa é imprescindível na avaliação energética desses materiais, uma vez que altos teores de cinza contribuem para a redução do poder calorífico, visto que os materiais minerais não participam do processo de combustão. Altos teores de cinzas em combustíveis podem resultar em emissão de partículas através das chaminés de caldeiras, tornando necessária a instalação de equipamentos para separação de partículas dos gases da combustão (BRAND, 2010).

Para a cama de aviário observa-se um elevado teor de cinzas, 20,05\%, quando comparado com as demais biomassas. Brand (2010) obteve teor de cinzas de $23,4 \%$, teor de carbono fixo de $13,87 \%$ e teor de voláteis de $62,73 \%$ para esse coproduto. A cama de aviário, por ser um material polidisperso e possuir outros materiais em sua composição além de serragem, tais como dejetos, penas de aves e ração, apresenta um valor inferior de carbono e elevado teor de cinzas (NEITZKE, 2010).

O valor de cinzas encontrados no presente trabalho foi inferior ao da literatura, já para carbono fixo e voláteis não apresentou grande variação. Essa diferença na composição, se deve às diferenças de regiões a qual foi coletada a amostra de cama de aviário, assim como a quantidade de lotes da cama, ou seja, a quantidade de vezes que a maravalha foi aproveitada.

Os valores encontrados na análise elementar (porcentagens de carbono, hidrogênio e oxigênio nas amostras são apresentados na Tabela 4. 
Tabela 4 - Análise elementar das amostras.

\begin{tabular}{cccc}
\hline Amostra & Hidrogênio (\%) & Carbono (\%) & Oxigênio (\%) \\
\hline BC & 5,75 & 45,74 & 42,79 \\
BM & 5,94 & 47,91 & 44,01 \\
CA & 4,80 & 39,27 & 35,32 \\
\hline
\end{tabular}

Fonte: Os autores, 2020.

Foi possível observar que o bagaço de mandioca (BM) apresentou maior teor de carbono $(47,91 \%)$ e hidrogênio $(5,94 \%)$ produzindo assim maior quantidade de energia quando queimado, comparado aos outros coprodutos. Ao comparar com a literatura, Zanatta (2012) encontrou os seguintes teores: carbono 40,4\%, hidrogênio 6,8\% e oxigênio 52 , $8 \%$ para bagaço de mandioca. Os valores encontrados são próximos aos valores da literatura.

O bagaço de cana-de-açúcar apresentou relação $\mathrm{C} / \mathrm{H}$ similar ao bagaço de mandioca, se mostrando um coproduto com elevada quantidade de energia liberada quando queimado. Em estudo realizado por Zanatta (2012), os teores encontrados para bagaço de cana foram 22,6\% para o teor de carbono, 2,7\% para o teor de hidrogênio e $74,6 \%$ de oxigênio. Já Ramos e Paula (2010) encontraram 45,3\% para o teor de Carbono, 6,8\% para Hidrogênio e $47,1 \%$ para Oxigênio. Pode-se observar que houve menor divergência entre o segundo autor citado e os experimentos realizados.

A cama de aviário apresentou baixo teor de hidrogênio (4,79\%), fato indesejável quando se visa a produção de energia, pois pequenos acréscimos no teor de hidrogênio promovem elevado ganho no valor calórico dos combustíveis de biomassa. Assim, compostos orgânicos que contenham maiores teores de carbono e hidrogênio liberam maior quantidade de energia quando queimados. Neitzke (2010), encontrou valores médios de 5,26\% para hidrogênio, 37,53\% para teor de carbono e 35,69\% para teor de oxigênio na cama de aviário, sendo esses valores muito próximos aos encontrados no presente trabalho.

\subsection{DETERMINAÇÃO DO PODER CALORÍFICO APÓS SECAGEM}

$\mathrm{Na}$ Tabela 5 são apresentados os valores de poder calorífico superior (PCS), poder calorífico inferior $(\mathrm{PCl})$ e poder calorífico útil (PCU) das amostras após secagem. 
Tabela 5 - Poder calorífico após secagem.

\begin{tabular}{cccc}
\hline Amostra & PCS (MJ/kg) & PCl $\mathbf{( M J} / \mathbf{k g})$ & PCU $\mathbf{( M J / k g )}$ \\
\hline BC & 17,52 & 16,22 & 15,21 \\
BM & 18,69 & 17,35 & 15,10 \\
CA & 15,45 & 14,36 & 14,14 \\
\hline
\end{tabular}

Fonte: Os autores, 2020.

O fator que tem maior influência sobre o poder calorífico é a umidade, pois essa influencia negativamente na quantidade de calor liberado durante a queima. Dessa forma, dentre as principais vantagens dos combustíveis densificados quando comparados a lenha está o poder calorífico elevado devido a menor umidade. O resultado é uma maior quantidade de energia por volume, reduzindo pátio de estocagem e equipamentos de queima (LIMA et al., 2008).

Para bagaço de mandioca (BM) o poder calorífico superior encontrado foi de 18,69 $\mathrm{MJ} / \mathrm{kg}$, o maior valor dentre os coprodutos analisados. Pode-se dizer que o bagaço de mandioca se apresenta como uma excelente fonte de energia, quando comparado a outros tipos de biomassas. Alves (2014) em seu estudo sobre o potencial energético de resíduos de produção agrícola, após briquetagem obteve poder calorífico superior de 16,73 MJ/kg para o bagaço de mandioca, $18,89 \mathrm{MJ} / \mathrm{kg}$ para maniva de mandioca, 18,92 $\mathrm{MJ} / \mathrm{kg}$ para sabugo de milho e 18,44 MJ/kg para palha de milho.

Para o bagaço de cana (BM) foi encontrado um poder calorífico superior de 17,52 $\mathrm{MJ} / \mathrm{kg}$. Em estudo semelhante Zanatta (2012) encontrou valor de PCS de 16,10 MJ/kg. Brand (2010) encontrou o valor de 16,31 MJ/kg. Marozzi (2012) encontrou valores similares de poder calorífico superior $(17,89 \mathrm{MJ} / \mathrm{kg})$. O valor encontrado mostrou como o bagaço de cana pode ser uma excelente fonte de energia.

O poder calorífico superior encontrado para cama de aviário foi de $15,45 \mathrm{MJ} / \mathrm{kg}$. Brand (2010) encontrou valor de PCS de 14,85 MJ/kg. O valor encontrado foi o menor dentre os coprodutos estudados, em função da composição química do material, principalmente ao elevado teor de cinzas $(20,05 \%)$ e também às diferentes condições de regiões, coleta e armazenamento do coproduto. 


\subsection{BRIQUETAGEM}

As misturas M1, M2 e M3 antes do processo de briquetagem são apresentadas na Figura 1. Na sequência foi feita a moagem das misturas.

Figura 1 - Misturas antes da briquetagem.

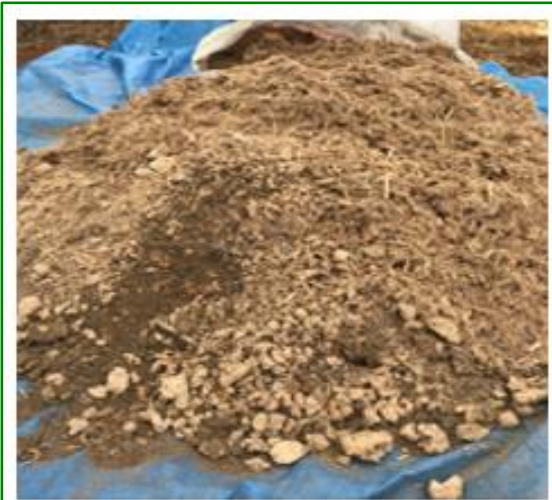

M1

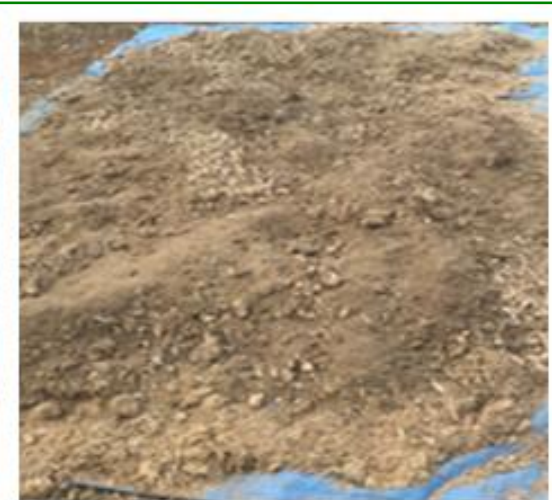

$\mathrm{M} 2$

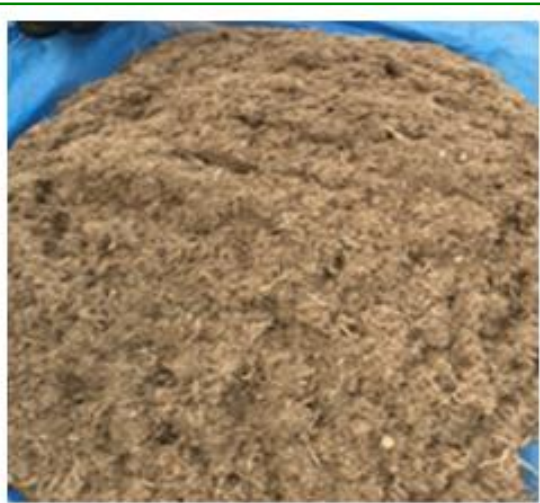

M3

Fonte: Os autores, 2020.

Para o processo de produção dos briquetes, a matéria-prima foi compactada em uma briquetadeira do tipo "rosca sem fim", sendo os briquetes fabricados com um orifício ao centro. Os briquetes apresentam o diâmetro de $67 \mathrm{~mm}$ e um espaço vazio no centro de 25 mm de diâmetro. Os briquetes M1, M2 e M3 podem ser observados na Figura 2. Essas medidas são características do equipamento utilizado.

Figura 2 - Briquetes produzidos.

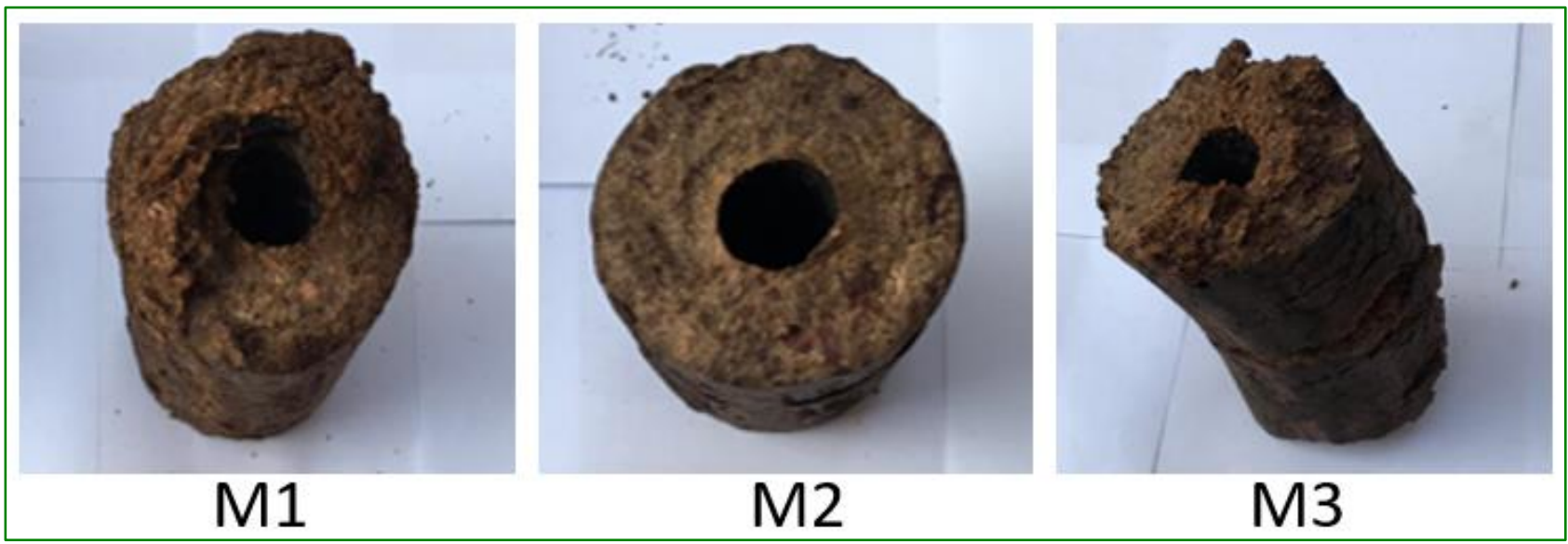

Fonte: Os autores, 2020. 


\subsection{ANÁLISE IMEDIATA PARA OS BRIQUETES PRODUZIDOS}

Os briquetes foram submetidos a análise imediata para determinação dos teores de umidade, carbono fixo, materiais voláteis e cinzas. Os resultados são apresentados na Tabela 6.

Tabela 6 - Valores médios da análise imediata.

\begin{tabular}{ccccc}
\hline Mistura & $\mathbf{U}(\%)$ & CF (\%) & MV (\%) & CZ (\%) \\
\hline M1 & $2,85(\mathrm{a})$ & $14,33(\mathrm{a})$ & $65,35(\mathrm{a})$ & $13,64(\mathrm{~b})$ \\
M2 & $4,78(\mathrm{~b})$ & $14,23(\mathrm{a})$ & $75,46(\mathrm{a})$ & $7,96(\mathrm{a})$ \\
M3 & $4,54(\mathrm{~b})$ & $15,04(\mathrm{a})$ & $72,84(\mathrm{a})$ & $10,77(\mathrm{a})$ \\
\hline \multicolumn{4}{c}{ * Letras iguais na coluna não diferem entre si, pelo teste de Tukey a 5\% de probabilidade. } \\
\multicolumn{5}{c}{ Fonte: Os autores, 2020. }
\end{tabular}

Aplicando-se o teste de Tukey a 5\% de significância foi possível concluir que os briquetes M2 e M3 não apresentaram diferenças estatísticas nos parâmetros umidade, carbono fixo, voláteis e cinzas. Já o briquete M1 apresentou diferenças estatísticas no teor de umidade (U\%) e cinzas (CZ\%) quando comparado com M2 e M3. Isso se deve ao elevado teor de cama de aviário (65\%) utilizado na mistura M1, influenciando diretamente nos parâmetros umidade (U\%) e cinzas (CZ\%). Também foi observado menor relação de carbono fixo (CF\%) e materiais voláteis (MV\%) para essa mistura (M1). Para os teores de materiais voláteis (MV\%) e carbono fixo (CF\%) não houve diferença estatística entre os briquetes produzidos.

Segundo Gonçalves, Sartori e Leão (2009) o teor de voláteis indica a porcentagem de materiais com potencial de geração de calor e o teor de cinzas corresponde à porcentagem de material que não produz calor. Portanto, o teor de voláteis e o teor de cinzas fornecem informações aproximadas da combustibilidade dos resíduos, sendo um fator importante para o processo de briquetagem.

Podemos observar que a mistura M2 apresentou menor teor de carbono fixo $(14,23 \%)$ maior teor de voláteis $(75,46 \%)$ e o menor teor de cinzas $(7,96 \%)$. Isso ocorre devido maior teor de bagaço de mandioca (15\%) comparado às outras misturas. Por apresentar alto poder calorífico facilita a combustão do material e eleva a temperatura. Já o teor de cinzas foi o menor encontrado em função da composição química do coproduto.

A mistura M1 apresentou menor valor de voláteis (65,35\%), em contrapartida possui maior valor de teor de cinzas (13,64\%). Esses valores podem estar associados a 
quantidade de cama de aviário utilizado na mistura, sendo que o mesmo já possui essas características individualmente quando comparado aos outros materiais utilizados.

Ao analisarmos outros estudos realizados pela literatura, Protásio et al. (2011) produziram briquetes a partir de resíduos do processamento de grãos de café submetidos aos processos de carbonização e torrefação, e obtiveram valores de $22 \%, 69 \%$ e $8 \%$ de teor de materiais voláteis, teor de carbono fixo e teor de cinzas, respectivamente, para briquetes carbonizados. Pode-se observar que os valores encontrados para briquetes carbonizados são distintos dos encontrados no presente trabalho, apresentando menor teor de materiais voláteis e maior teor de carbono fixo, em função do processo de carbonização.

Em pesquisa realizada por Souza et al. (2015) foram analisados briquetes produzidos com $40 \%$ de serragem de madeira e $60 \%$ de borra de café, que apresentaram teor de voláteis de $85,84 \%$ e teor de cinzas de $5,17 \%$. Pode-se observar valores mais próximos aos encontrados no presente estudo, ainda que um teor de cinzas inferior ao entrado na presente pesquisa.

\subsection{ANÁLISE ELEMENTAR PARA OS BRIQUETES PRODUZIDOS}

As porcentagens de carbono, hidrogênio e oxigênio na composição dos briquetes foram estimadas e são apresentadas na Tabela 7.

Tabela 7 - Valores médios da análise elementar.

\begin{tabular}{cccc}
\hline Mistura & H (\%) & C (\%) & O (\%) \\
\hline M1 & $4,95(\mathrm{~b})$ & $40,13(\mathrm{~b})$ & $36,63(\mathrm{~b})$ \\
M2 & $6,89(\mathrm{a})$ & $55,52(\mathrm{a})$ & $51,06(\mathrm{a})$ \\
M3 & $5,89(\mathrm{ab})$ & $47,50(\mathrm{ab})$ & $43,62(\mathrm{ab})$ \\
\hline
\end{tabular}

* Letras iguais na coluna não diferem entre si, pelo teste de Tukey a 5\% de probabilidade.

Fonte: Os autores, 2020.

Aplicando-se o teste de Tukey a 5\% de significância foi possível concluir que os briquetes M1, M2 e M3 apresentaram diferenças estatísticas entre si, nos parâmetros Hidrogênio, Carbono e Oxigênio. As variações nas misturas dos coprodutos influenciaram diretamente na composição.

A mistura M2 é a que possui maior percentual de C (55,52\%) e H (6,89\%), sendo assim, possui maior potencial energético na queima por apresentar maior proporção de bagaço de mandioca em sua mistura. Já para M1 o teor de carbono $(40,13 \%)$ e hidrogênio 
$(4,95 \%)$ foram os menores encontrados, devido ao maior teor de cama de aviário $(65 \%)$, reduzindo o potencial energético da mistura. Conforme Protásio et al. (2011) compostos orgânicos que contenham teores de carbono e hidrogênio maiores, possuem maior quantidade de energia quando queimados.

\subsection{DETERMINAÇÃO DO PODER CARÍFICO PARA OS BRIQUETES RODUZIDOS}

Foi estimado o poder calorífico superior, inferior e útil dos briquetes e os valores médios são apresentados na Tabela 8.

Tabela 8 - Valores médios de Poder Calorífico dos briquetes.

\begin{tabular}{cccc}
\hline Mistura & $\mathbf{P C S}(\mathbf{M J} / \mathbf{k g})$ & $\mathbf{P C l}(\mathbf{M J} \mathbf{k g})$ & $\mathbf{P C U}(\mathbf{M J} \mathbf{k g})$ \\
\hline M1 & $13,88(\mathrm{a})$ & $12,89(\mathrm{a})$ & $12,42(\mathrm{a})$ \\
M2 & $16,41(\mathrm{a})$ & $15,22(\mathrm{a})$ & $14,39(\mathrm{a})$ \\
M3 & $16,85(\mathrm{a})$ & $15,63(\mathrm{a})$ & $14,77(\mathrm{a})$ \\
\hline \multicolumn{2}{r}{${ }^{*}$ Letras iguais na coluna não diferem entre si, pelo teste de Tukey a 5\% de probabilidade. }
\end{tabular}

Fonte: Os autores, 2020.

Aplicando-se o teste de Tukey a $5 \%$ de significância pode-se concluir que os briquetes M1, M2 e M3 não apresentaram diferenças estatísticas nos parâmetros poder calorífico superior, inferior e útil (devido a elevada variação encontrada nas repetições dos tratamentos), sendo assim os briquetes podem ser considerados igualmente vantajosos do ponto de vista energético.

Resultados encontrados na literatura mostram que Silva et al. (2018) produziu briquetes utilizando aglutinante de amido de milho com PCS de $18,56 \mathrm{MJ} / \mathrm{kg}$ e com aglutinante de papel com PCS de 18,11 MJ/kg. Martins et al. (2016) ao analisar briquetes feitos a partir de finos de carvão vegetal compactados com resíduo celulósico e amido de milho, encontrou valores de PCS de 19,62 MJ/kg para os briquetes com resíduo celulósico e de $18,30 \mathrm{MJ} / \mathrm{kg}$ para os briquetes contendo amido.

Protásio et al. (2011), realizou a compactação de briquetes de serragem de eucalipto e obteve poder calorífico superior de $18,46 \mathrm{MJ} / \mathrm{kg}$ e também realizou a compactação de briquetes de resíduos de milho e obteve poder calorífico superior de 18,89 MJ/kg. Valores esses maiores que os encontrados no presente trabalho para M1, M2 e M3, mostrando assim que a utilização de matéria prima de origem florestal contribui no aumento do poder calorífico do briquete. 
Ao observarmos resultados encontrados pela empresa TEC - Tecnologia em Calor Ltda, podemos comparar os valores de poder calorífico inferior da lenha com $40 \%$ de umidade, de 10,04 MJ/kg e lenha seca (12\% de umidade), que apresentou 15,39 MJ/kg. Podemos observar que a lenha seca apresentou valor próximo de poder calorífico inferior para M2 e M3, e valor inferior a M1. Já quando comparamos com a lenha com 40\% de umidade, observamos que os briquetes produzidos apresentaram para as misturas M1, M2 e M3 valores superiores de poder calorífico inferior.

\subsection{DENSIDADE APARENTE E DENSIDADE ENERGÉTICA}

Os valores obtidos na análise de densidade aparente e energética dos briquetes são apresentados na Tabela 9. A densidade energética foi calculada multiplicando-se o poder calorífico superior pelos seus valores de densidade aparente.

Tabela 9 - Densidade aparente e densidade energética dos briquetes.

\begin{tabular}{|c|c|c|}
\hline Mistura & Densidade aparente $\left(\mathrm{kg} / \mathrm{m}^{3}\right)$ & Densidade energética $\left(\mathrm{MJ} / \mathrm{m}^{3}\right)$ \\
\hline M1 & $1230,57(a)$ & $17080,31(b)$ \\
\hline M2 & $1240,88(a)$ & $20362,84(a)$ \\
\hline M3 & $1153,25(b)$ & 19432,26(a) \\
\hline Desvio padrão & 36,65 & 1252,10 \\
\hline
\end{tabular}

A densidade aparente dos briquetes no Brasil, segundo Gentil (2008) varia de 1.000 $\mathrm{kg} / \mathrm{m}^{3}$ a $1.400 \mathrm{~kg} / \mathrm{m}^{3}$. Os resultados encontrados para M1, M2 e M3 ficaram dentro dos valores citados. Pimenta et al. (2015) encontrou valor de densidade aparente entre 660 $\mathrm{kg} / \mathrm{m}^{3}$ e $710 \mathrm{~kg} / \mathrm{m}^{3}$ para briquetes fabricados a partir de carvão de cascas de coco utilizando amido e argila como aglutinante, sendo valores inferiores aos encontrados no presente trabalho.

Podemos observar que 0 briquete M2 apresentou densidade aparente estatisticamente igual a M1, e densidade energética estatisticamente igual a $M 3$, sendo estes os maiores valores, o que pode ser justificado pelo maior teor de bagaço de mandioca, que em análise in natura apresentou elevado poder calorífico além de ser utilizado como material ligante pelo rico teor de amido. A amostra M1 apresentou elevada densidade aparente, porém em contrapartida um valor inferior de densidade energética devido ao baixo poder calorífico encontrado em sua mistura. A mistura M3 apresentou a menor 
densidade aparente, porém sua densidade energética foi maior que a M1 por apresentar poder calorífico elevado, maior teor de bagaço de cana e menor teor de cama de aviário.

\subsection{ANÁLISE DE COMPRESSÃO PARA OS BRIQUETES PRODUZIDOS}

As Figuras 3, 4 e 5 ilustram os resultados do teste de compressão nos briquetes M1, M2 e M3, apresentando suas respectivas forças máxima ( $\mathrm{N})$ e tensão máxima ( $\mathrm{N} / \mathrm{mm}$ $\left.\mathrm{kgf} / \mathrm{cm}^{2}\right)$, e também os briquetes sendo submetidos à análise.

Figura 3 - Análise de compressão briquete M1.

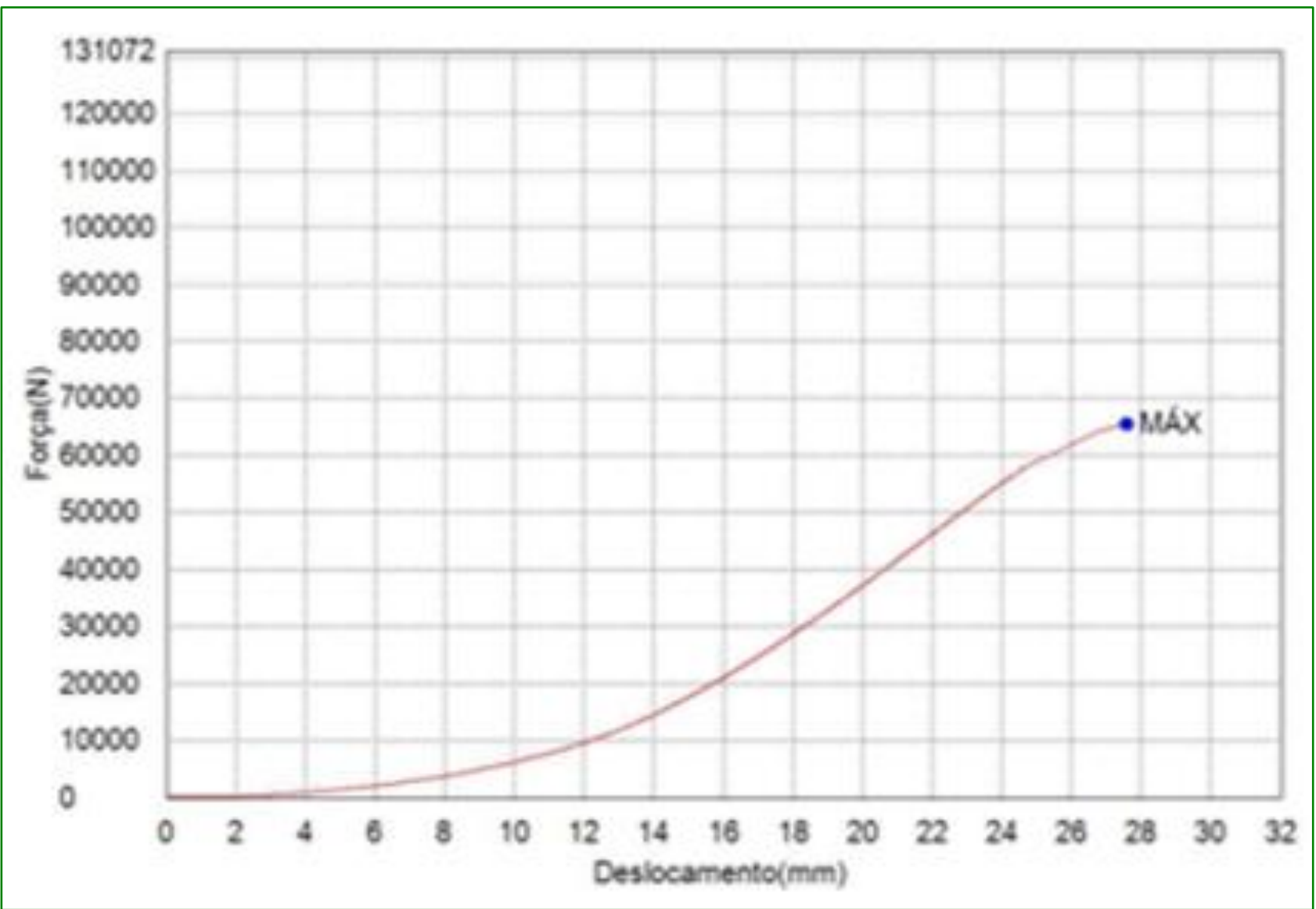

Fonte: Os autores, 2020. 
Figura 4 - Análise de compressão briquete M2.

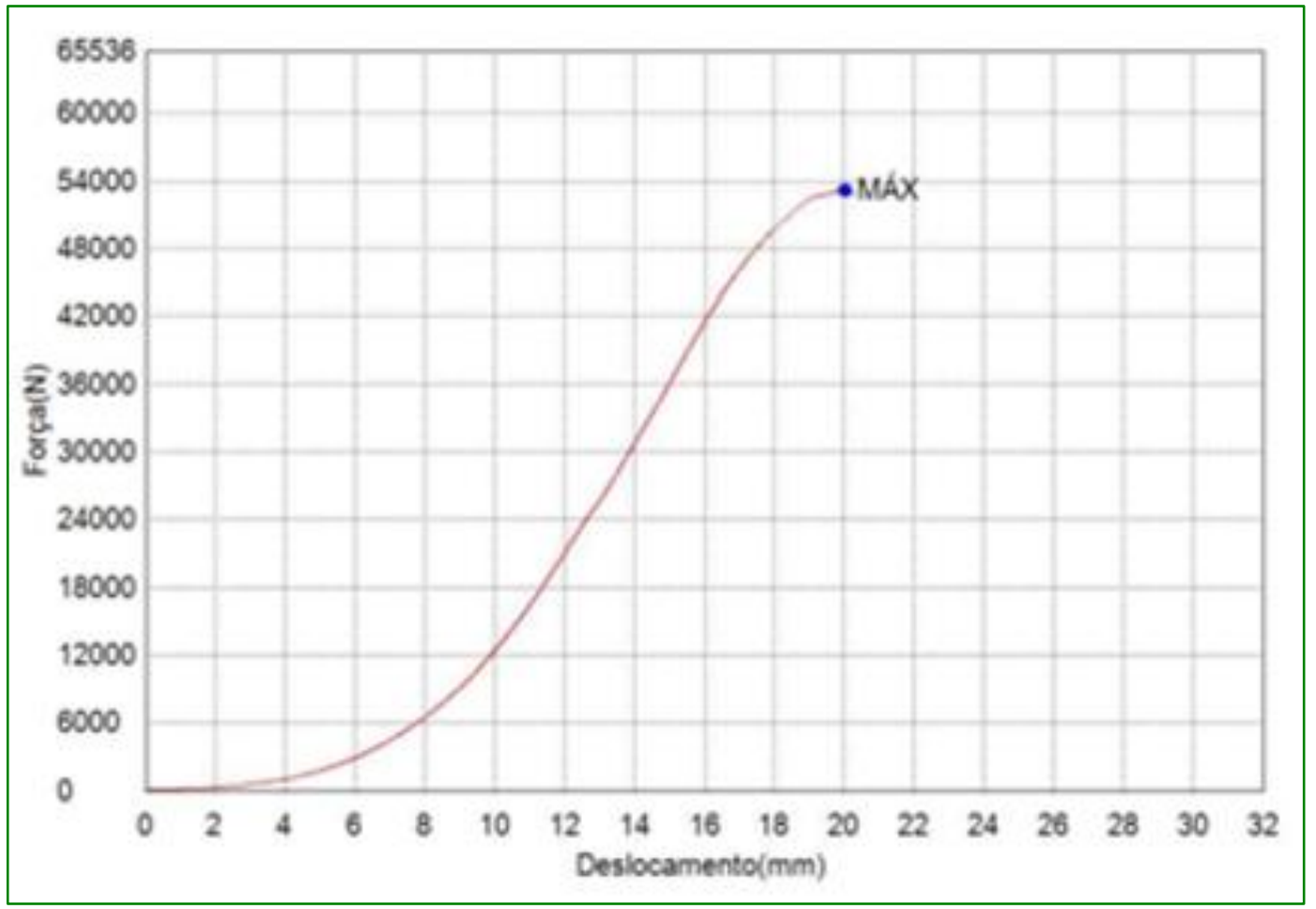

Fonte: Os autores, 2020.

Figura 5 - Análise de compressão briquete M3.

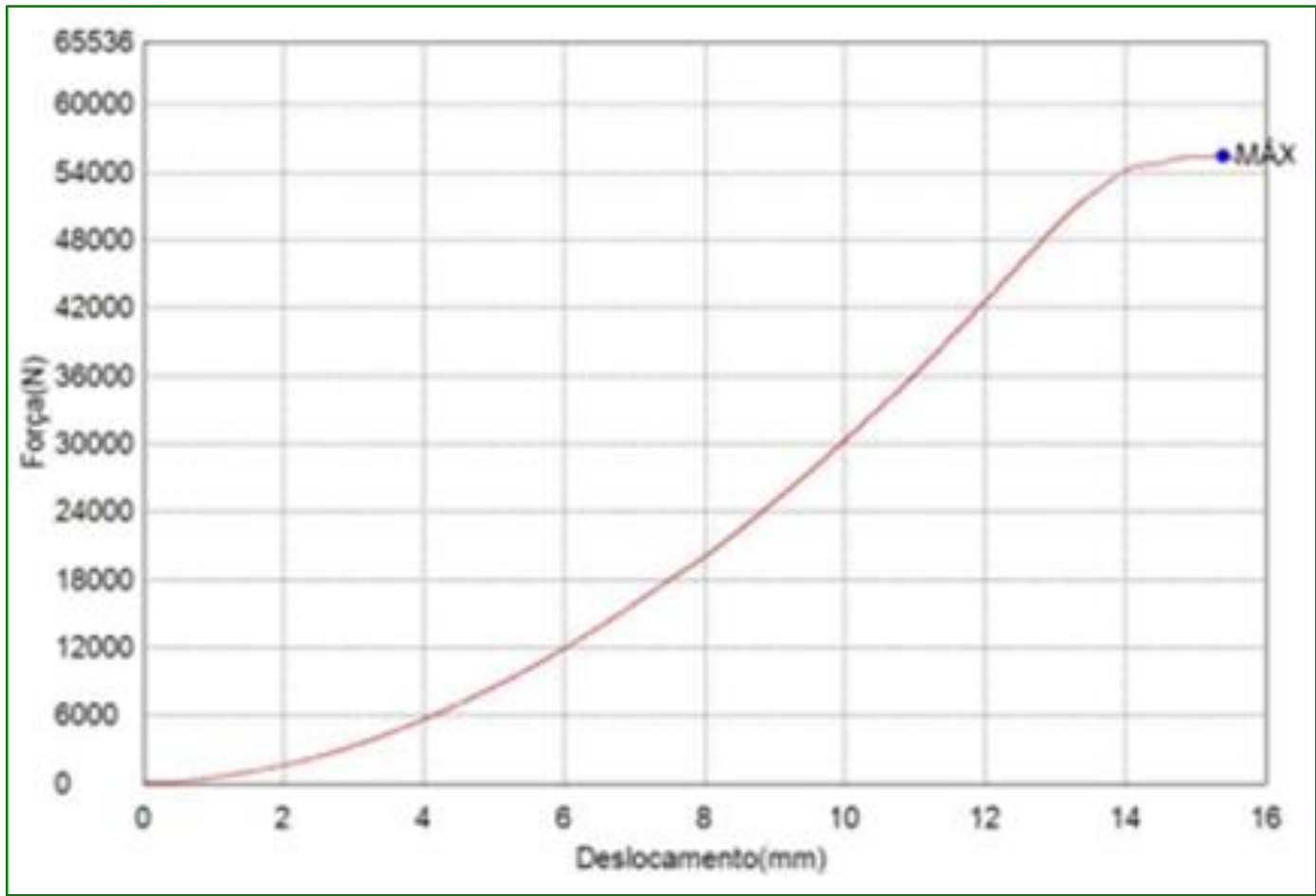

Fonte: Os autores, 2020. 
Os resultados obtidos no teste de compressibilidade são apresentados na Tabela 10.

Tabela 10 - Análise de compressibilidade.

\begin{tabular}{|c|c|c|c|c|}
\hline Mistura & Força Máx. (N) & Tensão Máx. (N/mm²) & Tensão Máx. (Kgf/cm²) & Deslocamento (mm) \\
\hline M1 & 65579,00 & 18,15 & 185,09 & 28 \\
\hline M2 & 53125,00 & 14,70 & 149,94 & 20 \\
\hline M3 & 55464,10 & 15,35 & 156,54 & 15 \\
\hline Média & 58056,00 & 16,06 & 163,86 & 21 \\
\hline
\end{tabular}

Fonte: Os autores, 2020.

O briquete M1 apresentou maior resistência com uma força máxima e tensão máxima superior a outras amostras. Já as amostras M2 e M3 apresentaram semelhança no teste, apresentando diferenças apenas em seu deslocamento.

Teixeira et al. (2010) descrevem que briquetes com resistência mecânica superior a $71,38 \mathrm{kgf} / \mathrm{cm}^{2}$ são considerados bons, pois a partir dessa resistência, podem ser manipulados e armazenados sem que ocorra grande fragmentação. Dessa forma os briquetes M1, M2 e M3 apresentaram um valor de resistência consideravelmente bom.

Quirino e Brito (1991) encontraram resistência à compressão média de $31,71 \mathrm{kgf} / \mathrm{cm}^{2}$ em briquetes produzidos com carvão vegetal, serragem, carvão mineral, nitrato, cinzas e aglomerante, valor este inferior aos encontrados neste estudo. Fontes et al. (1984) encontraram 183,76 kgf/ $/ \mathrm{cm}^{2}, 245,74 \mathrm{kgf} / \mathrm{cm}^{2}$ e $229,70 \mathrm{kgf} / \mathrm{cm}^{2}$, para briquetes produzidos a partir de carvão vegetal e resina termoplástica sintética, resina termoplástica natural e resina termorrígida, respectivamente.

Percebe-se que a resistência mecânica dos briquetes é afetada pelo tipo de matériaprima, além das quantidades de umidade e aglutinante na composição. No presente trabalho não foi utilizado aglutinante artificial, apenas bagaço de mandioca que pode ser utilizado como aglutinante natural por possuir elevado teor de amido na sua composição.

\subsection{COMPARAÇÃO COM NORMAS INTERNACIONAIS}

O mercado europeu se destaca na normatização de briquetes e pellets, com objetivo de garantir a uniformidade do produto visando o benefício de seus consumidores (DIAS et al., 2012). As normas foram implementadas isoladamente na Alemanha, Áustria e Suécia, com normatizações individuais, apresentando assim diferenças em diversos parâmetros de qualidade. Essas divergências nos parâmetros dificultou 0 atendimento para países 
exportadores que visavam o mercado europeu. Dessa forma o Comitê Europeu de Normatização (CEN) promoveu a uniformização das normas em todo continente.

$\mathrm{Na}$ Alemanha visando a qualidade do produto, os parâmetros estabelecidos pelas normas são extremamente rigorosos estabelecendo valores máximos para eles. Na Tabela 11 são apresentados os parâmetros da Alemanha para produção de briquetes.

Tabela 11 - Padrão de qualidade para briquetes na Alemanha.

\begin{tabular}{|c|c|c|c|c|}
\hline & Umidade (\%) & Teor Cinzas (\%) & Densidade Partícula $\left(\mathbf{g} / \mathrm{cm}^{3}\right)$ & Poder Calorífico (MJ/kg) \\
\hline DNIN & $\leq 12$ & $\leq 0,7$ & $\geq 1,0$ & $\geq 16,5 a \geq 19,0$ \\
\hline M1 & 2,85 & 13,64 & 1,23 & 13,88 \\
\hline M2 & 4,78 & 7,96 & 1,24 & 16,41 \\
\hline M3 & 4,54 & 10,77 & 1,15 & 16,85 \\
\hline
\end{tabular}

Fonte: Adaptada de DIN CERTCO, 2015.

Ao compararmos os resultados encontrados com as normas da Alemanha, os briquetes M1, M2 e M3 atenderam aos parâmetros umidade e densidade da partícula. Para o poder calorífico apenas M3 atendeu ao parâmetro, sendo o único que está entre os teores exigidos na norma. Já para os valores de cinzas nenhum briquete atendeu a normatização alemã, pois todos estão acima de 0,7\%.

Na Tabela 12 são apresentados os padrões europeus para a produção de briquetes.

Tabela 12 - Padrão de qualidade para briquetes, certificação EN plus®.

\begin{tabular}{ccc}
\hline Propriedade & EN plus A1 & EN plus A2 \\
\hline Umidade $(\%)$ & $\leq 12$ & $\leq 15$ \\
Teor de cinzas $(\%)$ & $\leq 1,0$ & $\leq 1,5$ \\
Densidade de partículas $\left(\mathrm{g} / \mathrm{cm}^{3}\right)$ & $\geq 1,0$ & $\geq 0,9$ \\
Poder calorifico $(\mathrm{MJ} / \mathrm{kg})$ & $\geq 15,5$ & $\geq 15,3$ \\
\hline
\end{tabular}

Fonte: Adaptada de European Pellet Council; Associação Européia de Biomassa, 2015.

Ao comparar os valores obtidos dos briquetes produzidos no presente trabalho com a norma europeia, pode-se observar que os briquetes produzidos seguem a normatização para alguns parâmetros, sendo estes umidade e densidade das partículas. Já para o poder calorífico apenas M2 e M3 se encaixam nos padrões. Com relação ao teor de cinzas nenhum briquete possui resultado satisfatório quando comparado aos valores apresentados na norma acima. 


\section{CONCLUSÕES}

Os resultados encontrados para Bagaço de mandioca se mostraram interessantes por apresentarem um baixo teor de cinzas e elevado poder calorífico. Esse composto será primordial na formação dos briquetes, atuando como material aglutinante. Apesar disso, o material in natura apresenta teor de umidade acima de $85 \%$, necessitando de um processo de secagem robusto, gerando, consequentemente, maior gasto energético.

Para o bagaço de cana-de-açúcar, os teores encontrados foram satisfatórios, em relação a umidade e poder calorífico. $O$ teor de cinzas encontrado foi o menor comparado aos valores encontrados na literatura. A cama de aviário apresentou elevado teor de cinzas, próximo a $20 \%$, tornando duvidosa sua utilização para geração de energia com a produção de briquetes. Porém, visando o cuidado ambiental, em regiões de grande produção de frango, pode-se interessar o uso.

Em relação a resistência mecânica os briquetes M1, M2 e M3 apresentaram um valor de tensão máxima acima de $71,38 \mathrm{kgf} / \mathrm{cm}^{2}$, então são considerados de boa resistência. Quanto à densidade aparente e energética encontrada para os briquetes, foram encontrados valores coerentes com a literatura, sendo eles satisfatórios para geração de energia.

A mistura que obteve melhor eficiência energética foi o briquete produzido a partir da mistura M2, o qual apresentou um poder calorífico de 16,41 MJ/kg e também o menor teor de cinzas, de $7,96 \%$, e melhor relação $\mathrm{C} / \mathrm{H}$, porcentagem de hidrogênio e de carbono, sendo assim o de maior potencial energético de queima.

Muitas vantagens são encontradas no uso de briquetes produzidos a partir da biomassa residual, como menor custo, facilidade de transporte e armazenamento e redução de impacto ambiental, permitindo assim o aproveitamento de resíduos. Os resultados confirmam que sua utilização contribui em aspectos econômicos, sociais e ambientais, além de incentivar a utilização de bioenergia.

\section{REFERÊNCIAS}

ALI, N. U.; NINA, P. M.; TARLUE, P. J. V.; NAKANWAGI, R.; KUTIOTE, J. O. E.; NUR, A. A.; CHANDA, P. Assessment of biomass briquette use as alternative source of renewable energy in kampala district. African Journal of Environment and Natural Science Research. V.2 p.68-76, 2019. 
ALVES, D. A. H. Avaliação do potencial energético de resíduos de produção agrícola provenientes do beneficiamento da mandioca e do milho. Universidade Federal de Alagoas, 2014.

ASSOCIAÇÃO BRASILEIRA DE NORMAS TÉCNICAS. NBR 5739: Ensaio de compressão de corpos de prova cilíndricos. 2018.

BONASSA, G.; SCHNEIDER, L. T.; CANEVER, V. B.; CREMONEZ, P. A.; FRIGO, E. P.; DIETER, J.; TELEKEN, J. G. Scenarios and prospects of solid biofuel use in Brazil. Renewable and Sustainable Energy Reviews. V.82, p. 2365-2378, 2018.

BORGES, A. C. P.; SILVA, M. S.; ALVES, C. T.; TORRES, E. A. Energias renováveis: uma contextualização da biomassa como fonte de energia. REDE- Revista Eletrônica do PRODEMA. Fortaleza, Brasil, v.10, n.2, p.23-36, 2016.

BRAND, M. A. Energia de Biomassa florestal. Rio de Janeiro: Interciência, 2010. 114 p.

COSTA, S. C. Briquetes a partir de resíduos de biomassa agrícola. Programa de Pósgraduação em ciências ambientais, UNISUL. 2019.

DIAS, J. M. S. SANTOS, D. T.; BRAGA, M.; ONOYAMA, M. M.; MIRANDA, C. H. B.; BARBOSA, P; F; D.; ROCHA, J; D. Produção de briquetes e péletes a partir de resíduos agrícolas, agroindustriais e florestais. Brasília, DF: Embrapa Agroenergia, 2012. $132 \mathrm{p}$.

DIN CERTCO. Certification scheme wood briquettes. Berlim, Junho de 2015.

EMBRAPA. Empresa Brasileira de Pesquisa Agropecuária. Produção de energia com resíduos da agropecuária - 2015. Disponível em: <https://bit.ly/2yTpWsx>. Acesso em: 03/05/2020.

EPE. Empresa De Pesquisa Energética. Balanço Energético Nacional 2020: ano base 2019. Rio de Janeiro, EPE, 2020.

EPE. Empresa de Pesquisa Energética. Estudos da demanda de energia. 2016. Disponível em: <https://bit.ly/2WT6c03>. Acesso em: 03/05/2020.

EUROPEAN PELLET COUNCIL. Associação Européia de Biomassa. Manual EN plus parte 3 - Requisitos de Qualidade de Pellets. Bélgica, V 3.0, Agosto de 2015.

FONTES, P. J. P. de; QUIRINO, W. F.; PASTORE JUNIOR, F.; FILGUEIRAS, S. M. S. Aglutinante para briquetagem de carvão vegetal. Brasília: DPQ/IBDF, 1984.

GENTIL, L.V.B. Tecnologia e Economia do Briquete de Madeira. 2008. 195p.Tese (Doutorado em Engenharia Florestal), Universidade de Brasília, Brasília, 2008.

GOES, T. A energia que vem da cana-de-açúcar. Agencia Embrapa de Informações Tecnológicas, 2008. 
GONÇALVES, J.E.; SARTORI, M.M.P.; LEÃO. A.L. Energia de briquetes produzidos com rejeitos de resíduos sólidos urbanos e madeira de Eucalyptus grandis. Energia Agrícola e Ambiental, v.13, n.5, p.657-661, 2009.

IBGE. Produção Agrícola Municipal 2017. Disponível em: <https://biblioteca.ibge. gov.br/visualizacao/periodicos/66/pam_2017_v44_br_informativo.pdf>. Acesso em: 04/05/2020.

IEA Bioenergy. Sustainable international bioenergy trade: securing supply and demand 2015. 2015.

KIM, D.; LEE, K.; PARK, K. Y. Upgrading the characteristics of biochar from cellulose, lignin, and xylan for solid biofuel production from biomass by hydrothermal carbonization. Journal of industrial and engineering chemistry. V.42, p.95-100, 2016.

\section{LIMA, E. A.; ABDALA, E. M.; WENZEL, A. A. Influência da umidade no poder calorífico superior da madeira. Embrapa, 2008.}

MAROZZI, C, R, B. Caracterização de Resíduos Agroindustriais e Florestais Visando a Briquetagem. 28 p. Universidade Federal do Espírito Santo, Gerônimo Monteiro, ES, 2012.

MARTINS, M. P. et al. Produção e avaliação de briquetes de finos de carvão vegetal compactados com resíduo celulósico proveniente da indústria de papel e cellulose. Revista Árvore, Viçosa, v. 40, n. 1, 2016.

MONTAGNA, T. B. Levantamento e análise de técnicas para disposição e tratamento de dejetos de suínos e de aves em estabelecimentos rurais familiares. Unioeste. Francisco Beltrão, 2017.

NEITZKE, G. Geração elétrica distribuída a partir da gaseificação de pellets de cama de aviário. Universidade de Brasília, Departamento de Engenharia Mecânica, Brasília, 2010.

PARIKH, J.; CHANNIWALA, S.A.; GHOSAL, G.K. A correlation for calculating HHV from proximate analysis of solid fuels. Science Direct. 19 November 2004.

PIMENTA, A. S.; SANTOS, R. C.; CARNEIRO, A. C. O.; CASTRO, R. V. O. Utilização de resíduos de coco (Cocos nucifera) carbonizado para a produção de briquetes.

Ciência Florestal, Santa Maria, v. 25, n.1, p.137-144, 2015

PROTÁSIO, T. P.; ALVES, I. C. N.; TRUGILHO, P. F.; SILVA, V. O.; BALIZA, A. E. R. Compactação de biomassa vegetal visando à produção de biocombustíveis sólidos.

Pesquisa Florestal Brasileira, v.31, p.273-283, 2011.

PROTÁSIO, T. P.; BUFALINO, L.; TONOLI, G. H. D.; COUTO, A. M.; TRUGILHO, P. F.; GUIMARÃES JÚNIOR, M. Relação entre o poder calorífico superior e os componentes elementares e minerais da biomassa vegetal. Pesquisa Florestal Brasileira, Colombo, v. 31, n. 66, p.122-133, 2011. 
PATTIYA, A.; TITILOYE J. O.; BRIDGWATER, A. V. Evaluation of catalytic pyrolysis of cassava rhizome by principal component analysis. Fuel, v. 89, p. 244-253, 2010.

PAULA, L. E. R.; TRUGILHO, P. F.; NAPOLI, A.; BIANCHI, M. L. Characterization of residues from plant biomass for use in energy generation. Cerne, v.17, p.237-246, 2011.

QUIRINO, W. F.; BRITO, J.O. Características e índice de combustão de briquetes de carvão vegetal. Brasília: LPF/IBAMA, 1991. 18p.

RAMOS E PAULA, L, E. Produção e Avaliação de Briquetes de Resíduos Lignocelulósicos. 72 p. Dissertação apresentada ao Programa de Pós-Graduação em Ciência e Tecnologia da Madeira - Universidade Federal de Lavras, Lavras - MG, 2010.

SAWIN, J. L.; BHATTACHARYA, S. C.; GALAN, E. M.; McCRONE, A.; MOOMAW, W. R.; SONNTAG-O'BRIEN, V.; SVERRISSON, F.; CHAWLA, K.; MUSOLINO, E.; SKEEN, J.; MARTINOT, E. Renewables 2012 Global Status Report. Paris: REN21, 2012. Disponível em: <https://bit.ly/3bpsJak>. Acesso em: 03/05/2020.

SILVA, V, V.; MAGALHAES, D, S; SIQUEIRA, W, C. Poder calorífico de briquetes artesanais fabricados com diferentes tipos de aglutinantes. Instituto Federal do Norte de Minas Gerais, Campus Araçuaí, 2018.

SOARES, L. S.; MORIS, V. A. S.; YAMAJI, F. M.; PAIVA, J. M F. Utilização de Resíduos de Borra de Café e Serragem na Moldagem de Briquetes e Avaliação de Propriedades. Matéria. Rio de Janeiro, v. 20, n. 2, p. 550-560, 2015.

TEIXEIRA, S. R.; PENA, A. F. V.; MIGUEL, A. G. Briquetting of charcoal from sugarcane bagasse fly ash (scbfa) as an alternative fuel. Waste management, v.30, n.5, p.804-807, 2010.

VIEIRA, A.C.M. Caracterização da biomassa proveniente de resíduos agrícolas para geração de energia. 2012. 56p. Dissertação (Mestrado de Energia na Agricultura), Universidade Estadual do Oeste do Paraná, Cascavel, 2012.

ZANATTA, E. R. Estudo Cinético da Pirolise das biomassas: Bagaço de mandioca, Casca de soja e Bagaço de Cana. Unioeste. Toledo, 2012.

ZENATTI, D. C.; COSTA, A. A.; HERMES, E.; VILVERT, R. M.; DIAS, L. C. Quantificação de açúcares redutores em hidrolisado do bagaço da mandioca para uso na produção de metano. Revista gestão e sustentabilidade ambiental. Florianópolis, n. esp, p.616-627, 2015. 


\section{ABSTRACT}

The current world energy scenario is the search for cleaner fuels and new sources of renewable energy, with emphasis on the use of residual biomass. The present work seeks to evaluate the physical-chemical characteristics of briquettes produced from sugarcane bagasse, cassava bagasse and poultry litter. The by-products underwent analysis before and after briquetting. Briquettes with different mixture percentages (M1, M2 and M3) were produced. The briquettes produced presented values of energetic efficiency, mechanical resistance and apparent and energetic density consistent with values in the literature and international standards. Regarding the ash content, none of them fit in the European briquettes manufacturing parameters. The mixture with better energy efficiency was M2, with a calorific value of $16.41 \mathrm{MJ} / \mathrm{kg}$ and also the smallest ash content of $7.96 \%$ and the best $\mathrm{C} / \mathrm{H}$ ratio, hydrogen and carbon percentage, thus being the one with the largest burning energy potential. The use of briquettes produced from residual biomass presents many advantages, contributing to economic, social and environmental aspects.

Keywords: Energy. Solid biofuel. Residual biomass.

\section{RESUMEN}

El escenario actual energético mundial es de búsqueda por combustibles más limpios y nuevas fuentes de energía renovables, destacando en la utilización de biomasa residual. Este trabajo busca evaluar las características físico-químicas de briquetas producidas a partir do bagazo de la caña de azúcar, bagazo de mandioca y cama de aviario. Los subproductos pasaron por análisis antes y después al briquetado Se produjeron briquetas con diferentes porcentajes de mezcla (M1, M2 y M3). Las briquetas producidas presentaron valores de eficiencia energética, resistencia mecánica y densidad aparente y energética coherentes con valores de la literatura y de normativas internacionales. En relación a la cantidad de cenizas ningún de ellos se encuadra en los parámetros europeos de fabricación de briquetas. La mezcla con mejor eficiencia energética fue M2, con poder calorífico de $16,41 \mathrm{MJ} / \mathrm{kg}$ y también el más bajo nivel de cenizas, de $7,96 \%$, y mejor relación $\mathrm{C} / \mathrm{H}$, porcentaje de hidrógeno y de carbono, y por consiguiente el de más grande potencial energético de quema. La utilización de briquetas producidas a partir de biomasa residual presenta muchas ventajas, contribuyendo en aspectos económicos, sociales y ambientales.

Palabras clave: Energía. Biocombustible sólido. Biomasa residual. 


\section{LICENÇA DE USO}

Este é um artigo publicado em acesso aberto (Open Access) sob a licença Creative Commons Atribuição 4.0 Internacional (CC BY 4.0), que permite uso, distribuição e reprodução em qualquer meio, desde que o trabalho original seja corretamente citado. Mais informações em: http://creativecommons.org/licenses/by/4.0

\section{CONFLITO DE INTERESSES}

Os autores declaram que não há conflito de interesses neste trabalho.

\section{CONTRIBUIÇÕES AUTORAIS}

Jaqueline Zanovelli Nalevaiko: Responsável pela autoria do texto.

Paulo André Cremonez: Responsável pela revisão e análise de dados.

Joel Gustavo Teleken: Responsável pela revisão.

\section{FINANCIAMENTO}

O presente trabalho não contou com apoio financeiro.

\section{COMO REFERENCIAR}

NALEVAIKO, Jaqueline Zanovelli; CREMONEZ, Paulo André; TELEKEN, Joel Gustavo. Utilização de subprodutos agroindustriais na produção de briquetes. Revista Brasileira de Engenharia de Biossistemas (Tupã), v. 15, n. 1, p. 1-26, 2021. DOI:

http://dx.doi.org/10.18011/bioeng2021v15n1p1-26.

\section{RESPONSABILIBADE EDITORIAL}

Prof. Dr. Fernando Ferrari Putti ${ }^{1}$, Prof. Dr. Paulo Sérgio Barbosa dos Santos ${ }^{1}$, Prof. Dr. Eduardo Festozo Vicente ${ }^{1}$ e Prof. Dr. Diogo de Lucca Sartori ${ }^{1}$

${ }^{1}$ Universidade Estadual Paulista "Júlio de Mesquita Filho", FCE - Faculdade de Ciências e Engenharia, Tupã, SP, Brasil. 